\title{
The expression and functional roles of microRNAs in stem cell differentiation
}

\author{
Jiwon Shim, ${ }^{1, *} \mathcal{E}$ Jin-Wu Nam ${ }^{1,2, *}$ \\ ${ }^{1}$ Department of Life Science, College of Natural Sciences, ${ }^{2}$ Research Institute of Natural Sciences, Hanyang University, Seoul 04763, Korea
}

microRNAs (miRNAs) are key regulators of cell state transition and retention during stem cell proliferation and differentiation by post-transcriptionally downregulating hundreds of conserved target genes via seed-pairing in their $3^{\prime}$ untranslated region. In embryonic and adult stem cells, dozens of miRNAs that elaborately control stem cell processes by modulating the transcriptomic context therein have been identified. Some miRNAs accelerate the change of cell state into progenitor cell lineages - such as myoblast, myeloid or lymphoid progenitors, and neuro precursor stem cells - and other miRNAs decelerate the change but induce proliferative activity, resulting in cell state retention. This cell state choice can be controlled by endogenously or exogenously changing miRNA levels or by including or excluding target sites. This control of miRNA-mediated gene regulation could improve our understanding of stem cell biology and facilitate their development as therapeutic tools. [BMB Reports 2016; 49(1): 3-10]

\section{INTRODUCTION}

The biology of microRNAs (miRNAs), small regulatory noncoding RNAs of $\sim 22$ nt in length, has yielded substantial understanding of the diverse cellular processes underlying posttranscriptional gene regulation and extended the gene regulatory network (1-8). Although the biogenesis, turnover, and gene regulatory mechanisms of miRNAs have been investigated extensively in the last decade, we are still uncovering new players and further extending our biological knowledge of miRNAs in diverse biological contexts. In particular, many miRNA studies have focused on stem-cell-related processessuch as proliferation, differentiation, and stemness - and the

*Corresponding authors. Jiwon Shim, Tel: +82-2-2220-2615; Fax: +82-2-2298-0319; E-mail: jshim@hanyang.ac.kr, Jin-Wu Nam, Tel: +82-2-2220-2428; Fax: +82-2-2298-0319; E-mail: jwnam@ hanyang.ac.kr

http://dx.doi.org/10.5483/BMBRep.2016.49.1.217

Received 19 October 2015

Keywords: Adult stem cell, Differentiation, Embryonic stem cell, microRNA, Proliferation, Self-renewal control of miRNA expression level and miRNA-mediated gene regulation used to assess the functional roles of miRNAs in stem cell processes $(5,8)$.

Stem cells are a subset of cells that possess the dual capacity to replenish themselves by self-renewal and to differentiate into special types of daughter cells that function as components of bodily structure (21). During this unique division, also known as asymmetric division, stem cells reprogram daughter cells to specify their fate by modulating multiple regulatory mechanisms while simultaneously maintaining their own stemness. Therefore, reprogramming daughter cells is accompanied by concurrent changes in the gene expression profile both for turning off the self-renewal capacity and for turning on cell-fate specific genes. Since the first discovery of miRNAs, knowledge of their role in stem cell control has increased exponentially due to advances in stem cell research.

High-throughput sequencing technology enables simultaneous profiling of expression of miRNAs and their derivatives in diverse cell types, including stem cells (9-13), and during embryonic and developmental processes (14-16), leading to identification of miRNAs as molecular determinants in a certain cellular process, and discovery of their functional roles in that context. Simultaneously, global analysis of miRNA targets in the same cellular processes led to identification of molecular signatures; i.e., shortening and lengthening of the $3^{\prime}$ untranslated region (UTR), and effects on targeting in the proliferation (17-19) and differentiation (20) processes.

This review will summarize the expression signatures of miRNAs during the self-renewal and differentiation of embryonic and adult stem cells. It will also address new mechanistic advances in regulation of miRNA production and targeting and discuss the functional roles of miRNAs in stem cell differentiation. These recent advances and future studies in miRNA biology will provide alternative approaches to, and tremendous insight into, translational research and facilitate use of miRNAs in clinical applications.

\section{RESULTS AND DISCUSSION}

miRNA expression during stem cell differentiation

Studies of miRNAs during embryonic stem cell (ESC) differentiation have led to discovery of the changes in miRNA levels and the regulation of miRNA expression during this process. 
Recent studies reported that miRNAs play important roles in modulating ESC self-renewal and differentiation (22-26). The upregulation of some miRNAs that maintain the stemness and self-renewal of ESCs and the downregulation of miRNAs that inhibit self-renewal blocks differentiation into adult stem cells. For instance, the miR-200 family and miR-302/367 cluster and miR-372, which promote self-renewal of human embryonic stem cells, are downregulated; and let-7a, which suppresses self-renewal is upregulated, under the control of SOX2-Lin28 during differentiation to human neural stem cells (hNSCs) (Fig. 1) $(27,28)$. The downregulation of let-7 by Lin28 in ESCs led to upregulation of Trim71 that facilitates the G1-S transition to promote rapid ESC renewal $(27,29)$. Suppression of miR-200 family that targets ZEB2 represses the mesenchymal-to-epithelial transition (MET), whereas the overexpression of miR-200 family promotes the MET and induces pluripotent stem cell generation (30). Inhibition of ZEB2 mimicked the effects of the miR-200 family on pluripotent stem cell generation and the
MET (30). In addition, miR-302 and miR-372, ESC-specific cell cycle-regulating miRNAs, promote dedifferentiation of somatic cells by blocking TGF- $\beta$-induced epithelial-mesenchymal transition (EMT), by regulating the cell cycle and epigenetic state (31). The upregulation of the miR-302/367 cluster dually induced hESC self-renewal and blocked apoptosis by downregulating $\mathrm{BNIP} 3 \mathrm{~L} / \mathrm{Nix}$ and upregulating $\mathrm{BCL}-\mathrm{xL}$ expression $(22,32)$. Beside these miRNA clusters, many other miRNAs are dynamically changed during differentiation to NSCs, hepatocyte stem cells (HSCs), muscle stem cells (MSCs), and hematopoietic stem cells (HPSCs) $(5,8)$ (Fig. 1).

The expression of stem-cell differentiation-associated miRNAs is controlled by multi-layered regulatory processes including transcriptional, epigenetic, and/or post-transcriptional regulation. For instance, the miR-362/367 cluster is regulated by ESC-associated transcription factors (TFs), including Oct $3 / 4$, Sox 2 , and Rex1 (33), which induce pluripotent stem cell generation or self-renewal. Studies of transcriptional regulation of miRNA ex-

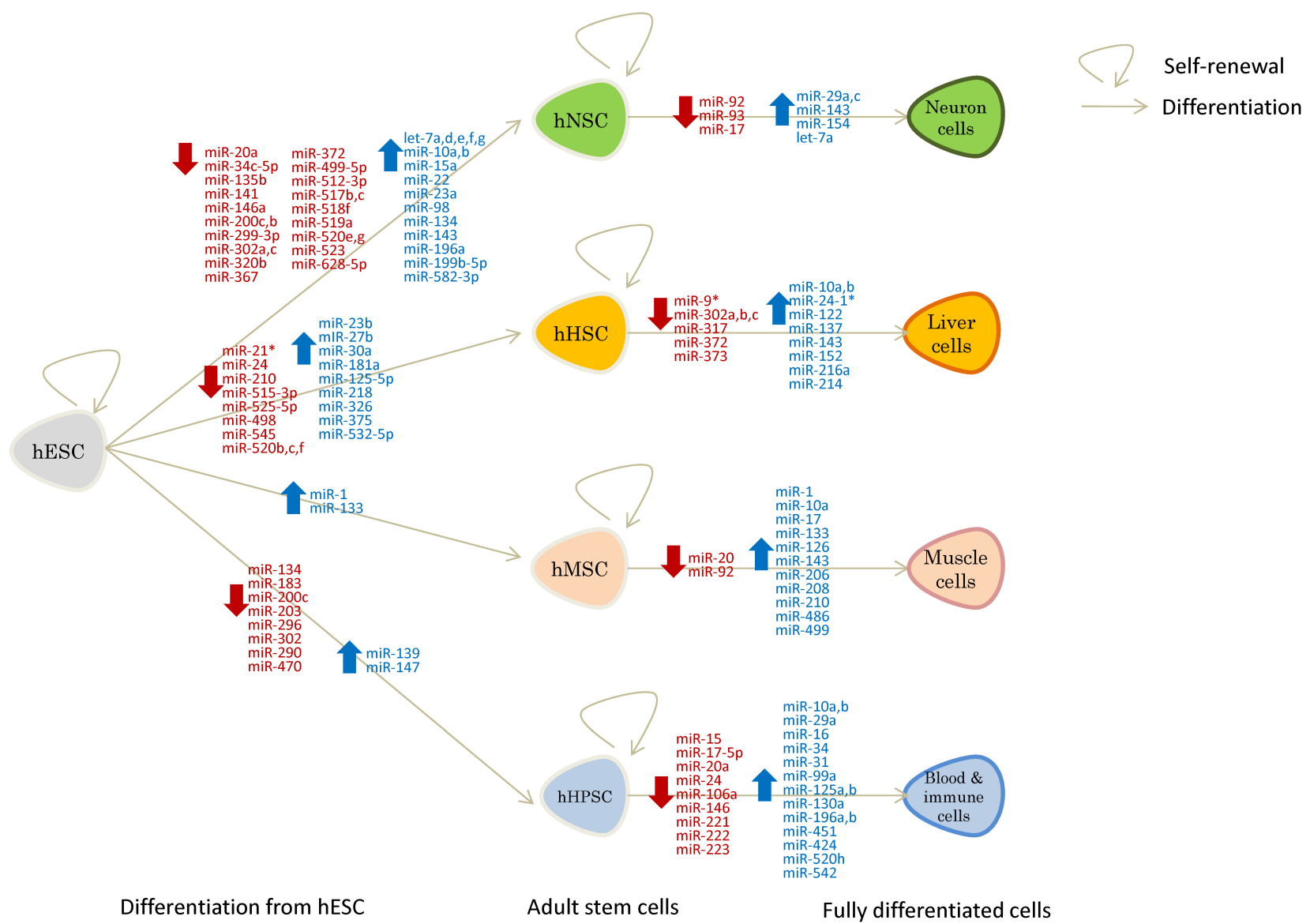

Fig. 1. miRNAs regulated during stem cell differentiation (blue: upregulated, red: downregulated). Shown are miRNAs up/downregulated during the differentiation of human embryonic stem cells (hESCs) to adult stem cells to fully differentiated cells. hNSCs: human neural stem cells; hHSCs: human hepatocyte stem cells; hMSCs: human muscle stem cells; hHPSC: human hematopoietic stem cells. A straight arrow indicates a differentiation process and a circular arrow indicates a self-renewal process. 
pression in ESCs have revealed that mono-cistronic or bi-cistronic miRNAs located at intergenic loci tend to be regulated by common upstream transcription regulators, whereas miRNAs located in intronic regions tend to be regulated by transcription regulators of the host gene. For example, mir-206, mir-486, and mir-499 and the bi-cistronic miRNA mir-1 and mir-133 are activated by MyoD, myogenin, and MYF5 during differentiation of human muscle stem cells (hMSCs) (34-38). Interestingly, the coherently regulated mir-1 and mir-206 in different chromosomes are members of a same miRNA family, and share a seed sequence that downregulates common targets (39).

The epigenetic regulation of chromatin structure is also involved in miRNA expression during ESC differentiation. Small-RNA sequencing (sRNA-seq) and Chromatin Immunoprecipitation followed by sequencing (ChIP-seq) of histone modification markers uncovered epigenetically modulated
miRNAs during lymphopoiesis. Lymphocyte-specific miRNAs are tightly regulated by PRC1-mediated a histone 3 lysine 27 tri-methylation (H3K27me3) (40). For instance, miR-139 and miR-147 were upregulated in ProB and PreB cells by reduction of H3K27me3, while miR-152 was downregulated in the cells by the gain of H3K27me3 (40). Another recent study demonstrated that a histone 3 lysine 9 demethylase (JMJD1C), expressed in hESCs, suppresses the neural differentiation of hESCs by epigenetically maintaining miR-302 expression, and downregulation of the JMJD1C is sufficient to induce neural differentiation (26).

Post-transcriptional regulation is generally used for fine-tuning of miRNA expression by controlling either the stability of precursor and mature miRNAs or the maturation process itself. The stability of pre-let-7 is regulated by lin28, which recruits 3' terminal uridylyl transferase (TUTase) to selectively suppress let-7 miRNA biogenesis in undifferentiated cells (41). TuT4 and

\section{A}

\section{Antagomir}

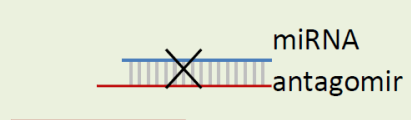

D

TALEN-based INDELS

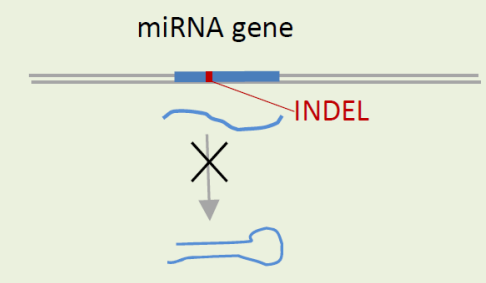

$\mathrm{H}$

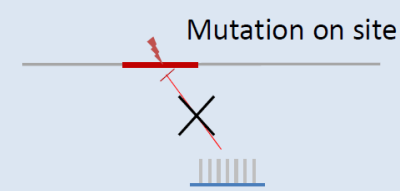

B miRNA sponge

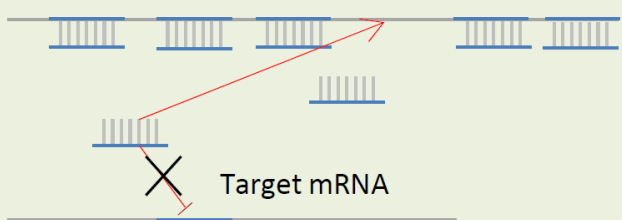

C HR-based knockout miRNA gene

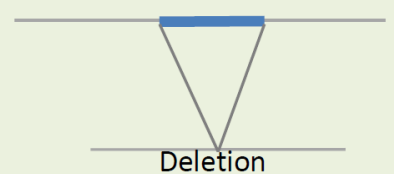

E

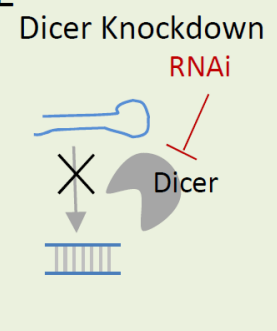

$\mathrm{F}$

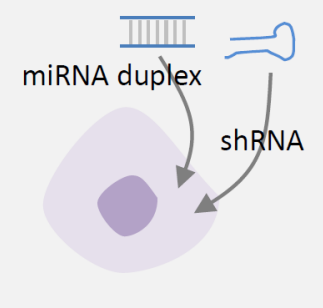

G

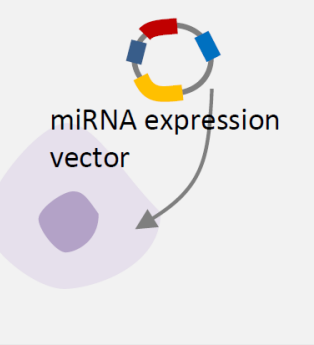

I

Inclusion by lengthening

exclusion by shortening

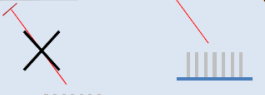

Fig. 2. Regulation of miRNA levels and target sites. (A-E) Loss-of-function approaches. (A) Antagomir directly suppresses an endogenous target miRNA. (B) miRNA sponge, which contains multiple target sites, quenches endogenous miRNAs. (C and D) miRNA knockout by homologous recombination $(\mathrm{C})$ and TALEN-mediated double-strand breaks $(\mathrm{D})$. ( $\mathrm{F}$ and $\mathrm{G})$ Gain-of-function approaches. (F) Introduction of short hairpin RNAs (shRNAs) or duplexes. (G) Introduction of miRNA expression vectors. (H and I) Target-side perturbation. (H) Site-directed mutagenesis of target site. (I) Alternative miRNA regulation by lengthening and shortening of 3'UTR. 
TuT7, which bind to lin28 via a zinc finger domain, redundantly uridylate pre-let-7, leading to downregulation of let-7 (41-43).

\section{Regulation of miRNA-mediated gene regulation}

The functional importance of miRNA during development and adult cell differentiation has been investigated by changing its expression or manipulating its target sites in embryonic and adult stem cells. Global downregulation of endogenous miRNAs by Dicer knockout or knockdown (Fig. 2E) in adult myogenic stem cells revealed that miRNAs are indispensable for proper myogenesis and myofiber morphology formation (44). Functional analysis of individual miRNAs has been performed by either miRNA-side perturbations-loss-of-functions (LOFs) and gain-of-functions (GOFs) of miRNAs (Fig. 2A-G) or target-side perturbations - mutation, inclusion, and exclusion of target sites in mRNA 3'UTRs (Fig. $2 \mathrm{H}$ and I). miRNA-side LOF is a straightforward approach to identifying the targets of an miRNA and to decipher its functional roles. First, antagomir (Fig. 1A), antisense RNA reverse-complementally binding to miRNA, and sponge (Fig. 1B), RNA with multiple sites predominantly quenching endogenous miRNAs, downregulate the level of endogenous miRNAs, leading to de-repression of their targets (45-48). Memczak et al. and Hansen et al. reported that an endogenous sponge RNA for miR-7 contains $>60$ conserved miR-7 seed sites, impairing miR-7-mediated gene regulation by absorbing the miR-7 RNAs in mouse neuronal tissues, which impaired midbrain development $(47,48)$.

An alternative miRNA-side LOF study is knockout of an miRNA gene in the genome by homologous recombination (HR)-based (Fig. 2C) or Transcription Activator-Like Effector Nucleases (TALEN)-based methods (Fig. 2D). Although HRbased knockout is a less-efficient approach, for the last decade, tens of HR-based miRNA knockouts have uncovered the functional roles of miRNAs in diverse biological processes, including development and differentiation (49). For instance, HRbased knockout of miR-144/451 impaired the late erythroblast maturation process (50) and knockout of miR-290-295 caused embryonic development delay and germ cell deficiency (51). Recently, more efficient TANEL-based knockout methods have been suggested $(52,53)$, and further miRNA knockout experiments will be performed in the context of embryo development and adult cell differentiation, enhancing our understanding of miRNA-mediated regulation.

In contrast to LOFs, miRNA-side GOFs can be performed by introducing miRNA duplexes, short hairpin RNAs (shRNAs), and miRNA expression vectors (Fig. 2F-G), which lead to downregulation of miRNA targets. Although the majority of the miRNA-side GOFs have been performed in cell lines, the lentiviral vector system has been frequently applied to ESCs to overexpress ESC-associated miRNAs. Xu et al. transduced a lentiviral vector containing mir-145 precursor to hESCs to examine the effect of miR-145 on endogenous OCT4, KLF4, and SOX2 in hESCs, and found reduction of OCT4 and KLF4, but not SOX2 expression (54). The overexpression of miR-145 was sufficient to inhibit hESC self-renewal (54). Lahmy et al. transduced a lentiviral vector containing human mir-375 precursor to hESCs and observed accelerated islet differentiation (55). More recently, Deng et al. introduced an miR-125b-2 lentiviral expression vector to $\mathrm{mESCs}$, and reported that miR-125b-2 inhibited the differentiation of $\mathrm{mESCs}$ into endoderm and ectoderm but did not affect their self-renewal or proliferation (56).

Because miRNA-side perturbation affects multiple biological processes through hundreds of conserved targets, it can be difficult to pinpoint target genes involved in the observed phenotypes. In contrast, target-side perturbation could less ambiguously verify a target gene and the functional role of the miRNA. Target site mutation (Fig. $2 \mathrm{H}$ ) and alternative polyadenylation mutation (Fig. 2l) enable assessment of the one-toone relationship between a miRNA and its target site by impairing their interaction. For instance, $\mathrm{Xu}$ et al. validated the direct regulation of OCT4, SOX2, and KLF4 using mutant $3^{\prime}$ UTR with a 6 bp deletion of the miR-145 target site (54). Lee et al. by introducing a point mutation at the target site demonstrated that miR-26a directly downregulates HDAC6 during myogenic differentiation of embryonic stem cells (57).

\section{Functional roles of miRNAs in adult stem cells}

miRNAs in hematopoiesis: Hematopoiesis comprises a series of proliferation and differentiation processes of blood cells originating from hematopoietic stem cells, which gives rise to complex cell types of the myeloid and lymphoid lineages (Fig. 3). A critical decision during hematopoiesis takes place during the differentiation of hematopoietic progenitors into either common lymphoid or common myeloid progenitors. A larger number of miRNAs have been identified in this complex process compared to other adult stem cells. There are three groups of miRNAs according to the means of hematopoietic stem cell control. 1) Some miRNAs are involved in hematopoietic stem/ progenitor control. For example, miR-128 and miR-181 maintain hematopoietic stem cells by inhibiting differentiation of all other hematopoietic lineages (58), while miR-16, miR-103 and miR-107 directly control the proliferative activities of the hematopoietic progenitors (58). 2) The other group of miRNAs specifies differentiation into the myeloid or lymphoid lineage from the hematopoietic progenitors. Representative examples of miRNA involved in lineage specification are miR-223 and miR-181, both of which exhibit low expression in hematopoietic stem cells but are highly upregulated during lineage-specific differentiation. 3) The third group comprises miRNAs important in the further differentiation of each lineage. Common myeloid progenitors (CMP) can be sub-divided into two precursors: granulocyte-macrophage precursors (GMP), also known as myeloblasts, and megakaryocyte-erythroid precursors (MEP), which give rise to megakaryocytes and erythroid cells through multiple steps of cell-type-specific differentiation. Studies have focused on the mechanisms underlying erythroid differentiation from the MEP due to its high 


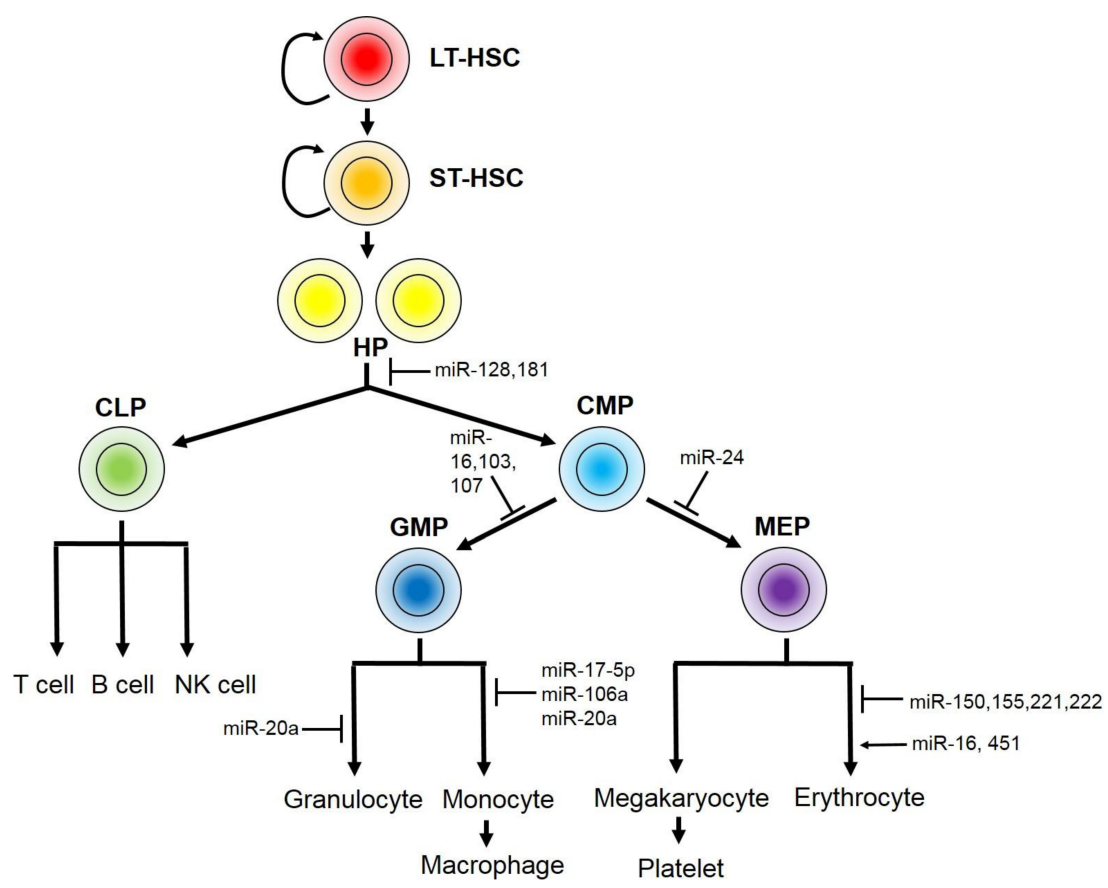

Fig. 3. miRNAs in hematopoiesis. Hematopoiesis comprises a series of proliferation and differentiation processes initiated from long-term hematopoietic stem cells (LT-HSC). LT-HSCs first give rise to short-term hematopoietic stem cells (ST-HSC), followed by differentiation to hematopoietic progenitors (HP). HPs differentiate into either common lymphoid progenitors (CLP) or common myeloid progenitors (CMP), which further differentiate to produce various types of blood cell. CLPs differentiate into lymphoid lineage blood cells, including $\mathrm{T}$ cells, B cells and NK cells. CMPs give rise to two restricted progenitors: granulocytemonocyte precursors (GMP) and megakaryocyte-erythrocyte precursors (MEP). The miRNAs that function in specific steps are indicated, and positive and negative controls are indicated by arrows and $\mathrm{T}$ lines, respectively. clinical value, and a large number of miRNAs involved in this process have been identified. Bioinformatics analysis has shown that miR-451 and miR-16 are upregulated during the late stage of erythropoiesis, whereas miR-150, miR-155, miR-221 and miR-222 are progressively downregulated during erythropoiesis, suggesting that they play functional roles during this process (59). Interestingly, miR-221 and miR-222 prevent erythroid differentiation by targeting KIT (also known as CD117), a cytokine receptor expressed in hematopoietic stem cells; indeed, downregulation of these miRNAs is essential for erythroid differentiation (60). In addition to the well-known erythrocyte growth factor, erythropoietin, activin promotes erythroid differentiation through its receptor ALK4, initiating a signaling cascade that culminates in erythroid differentiation. miR-24, which regulates ALK4 expression, therefore, serves as the gatekeeper for erythropoiesis (61). GMP gives rise to granulocytes - including basophils, neutrophils and eosinophils - and monocytes, which ultimately differentiate into macrophages. Monocyte differentiation is largely regulated by the master transcription factor AML1, which is targeted by miR-17-5p, miR-20a and miR-106a (62). In the lymphoid lineage, miR-150 is a key miRNA that enhances the differentiation of $T$ cells rather than B cells (63). In contrast, miR-181 induces lymphoid-lineage cell differentiation and ectopic expression of miR-181 in undifferentiated progenitor cells results in production of cells of the B lymphoid lineage (64).

miRNAs in myogenesis and cardiogenesis: Myogenesis includes multiple steps of proliferation and differentiation that require precise controls. Mesodermal stem/progenitor cells
(MSCs) first give rise to myoblasts, which later withdraw from the cell cycle and fuse into multinucleated myotubes (65). Specific miRNAs known as "myomiRs" play important roles in myogenesis (66-68). miR-1 and miR-133 are critical regulators of muscle cell proliferation and differentiation (69). miR-1 finetunes the balance between proliferation and differentiation of cardiac muscle cells by indirectly upregulating MEF2C, an essential muscle-related transcription factor, and by targeting HDAC4 (69). The myogenic function of miR-1 is conserved in Drosophila miR-1, indicative of universal myogenic activity of miR-1 in animals $(69,70)$. miR-133, on the other hand, targets serum response factor (SRF), an essential transcription factor involved in muscle differentiation (69). In human and mouse ES cells, both miR-1 and miR-133 activate mesoderm formation and inhibit expression of non-muscle genes. However, miR-1 counteracts miR-133 in cardiac progenitor formation (66). Another miRNA, miR-499, is enriched in cardiac progenitors and its overexpression accelerates the differentiation of beating embryoid bodies while repressing cardiac progenitor maintenance (67). miR-26a promotes skeletal muscle differentiation by targeting the histone methyl transferase enhancer of zeste homologue 2 (EZH2) (71). Expression of the miR-1792 cluster in adult cardiac progenitor cells leads to an increase in cardiac progenitor proliferation (72). In addition to the miRNA-mediated regulation of myogenic transcription factors, myogenic factors also regulate the expression of miRNAs. For example, SRF and the co-activator myocardin bind to the promoter of the mir-1 cluster, which increases the expression of primary mir-1 in cardiac progenitor cells (73). Another regu- 
lator of myogenesis, transforming growth factor $\beta$ (TGF $\beta$ ), suppresses miR-24 expression, which inhibits the expression of markers of myogenic differentiation (74).

miRNAs in the nervous system: A crucial lineage specification of the neural stem cell (NSC) takes place during the differentiation of neurons or astrocytes, one of the glial cell types. This process is regulated by distinct groups of miRNAs that mediate lineage-specific differentiation. Expression of miR-124 and miR-128 leads to the induction of neuronal cell fate (75). On the other hand, miR-124 targets the 3'UTR of SCP1, a small carboxy-terminal domain phosphatase 1 that binds to a conserved response element and suppresses the expression of neural genes, leading to astrocyte differentiation (76).

\section{Perspective}

Accompanied by recent progress in RNA biology and stem cell biology, critical roles of miRNAs in the maintenance and differentiation of stem cells have been revealed. Advances in deep-sequencing techniques and large-scale screening will lead to discovery of diverse functions of further miRNAs in various stem cell types. Recent studies have reported other types of non-coding RNAs, including groups of small non-coding RNAs and large non-coding RNAs, the functions of which have yet to be identified. It will be of interest to study the functions of such novel non-coding RNAs in stem cell control in addition to miRNAs, given the number of non-coding genes in the human genome. Elucidation of the biological mechanisms underlying miRNA-mediated control of stem cells will provide insight into how gene networks simultaneously orchestrate the expression of multiple target genes, which gives rise to precise consequences during development. Furthermore, these studies will be a basis for translational research and clinical application, as miRNAs possess tremendous potential for clinical applications and as drug targets.

\section{ACKNOWLEDGEMENTS}

This work was supported by the research fund of Hanyang University (HY-2012-2191) and by the "Cooperative Research Program for Agriculture Science \& Technology Development (Project No. PJ01045303)" of the Rural Development Administration, Republic of Korea.

\section{REFERENCES}

1. Arner P and Kulyte A (2015) MicroRNA regulatory networks in human adipose tissue and obesity. Nat Rev Endocrinol 11, 276-288

2. Bartel DP (2009) MicroRNAs: target recognition and regulatory functions. Cell 136, 215-233

3. Chen K and Rajewsky N (2007) The evolution of gene regulation by transcription factors and microRNAs. Nat Rev Genet 8, 93-103

4. Flynt AS and Lai EC (2008) Biological principles of microRNA-mediated regulation: shared themes amid diversity. Nat Rev Genet 9, 831-842

5. Gangaraju VK and Lin H (2009) MicroRNAs: key regulators of stem cells. Nat Rev Mol Cell Biol 10, 116-125

6. Ha M and Kim VN (2014) Regulation of microRNA biogenesis. Nat Rev Mol Cell Biol 15, 509-524

7. Pencheva $N$ and Tavazoie SF (2013) Control of metastatic progression by microRNA regulatory networks. Nat Cell Biol 15, 546-554

8. Shenoy A and Blelloch RH (2014) Regulation of microRNA function in somatic stem cell proliferation and differentiation. Nat Rev Mol Cell Biol 15, 565-576

9. Landgraf $P$, Rusu M, Sheridan R et al (2007) A mammalian microRNA expression atlas based on small RNA library sequencing. Cell 129, 1401-1414

10. Ahn HW, Morin RD, Zhao H et al (2010) MicroRNA transcriptome in the newborn mouse ovaries determined by massive parallel sequencing. Mol Hum Reprod 16, 463-471

11. Linsen SE, de Wit E, de Bruijn E and Cuppen E (2010) Small RNA expression and strain specificity in the rat. BMC Genomics 11, 249

12. Meunier J, Lemoine F, Soumillon M et al (2013) Birth and expression evolution of mammalian microRNA genes. Genome Res 23, 34-45

13. Hinton A, Hunter SE, Afrikanova I et al (2014) sRNA-seq analysis of human embryonic stem cells and definitive endoderm reveals differentially expressed microRNAs and novel IsomiRs with distinct targets. Stem Cells 32, 23602372

14. Ruby JG, Jan C, Player C et al (2006) Large-scale sequencing reveals 21U-RNAs and additional microRNAs and endogenous siRNAs in C. elegans. Cell 127, 1193-1207

15. Chiang HR, Schoenfeld LW, Ruby JG et al (2010) Mammalian microRNAs: experimental evaluation of novel and previously annotated genes. Genes Dev 24, 992-1009

16. Shkumatava A, Stark A, Sive H and Bartel DP (2009) Coherent but overlapping expression of microRNAs and their targets during vertebrate development. Genes Dev 23, 466-481

17. Sandberg R, Neilson JR, Sarma A, Sharp PA and Burge CB (2008) Proliferating cells express mRNAs with shortened 3 ' untranslated regions and fewer microRNA target sites. Science 320, 1643-1647

18. Mayr C and Bartel DP (2009) Widespread shortening of 3'UTRs by alternative cleavage and polyadenylation activates oncogenes in cancer cells. Cell 138, 673-684

19. Nam JW, Rissland OS, Koppstein D et al (2014) Global analyses of the effect of different cellular contexts on microRNA targeting. Mol Cell 53, 1031-1043

20. Boutet SC, Cheung TH, Quach NL et al (2012) Alternative polyadenylation mediates microRNA regulation of muscle stem cell function. Cell Stem Cell 10, 327-336

21. Hsu YC and Fuchs E (2012) A family business: stem cell progeny join the niche to regulate homeostasis. Nat Rev Mol Cell Biol 13, 103-114

22. Anokye-Danso F, Trivedi CM, Juhr D et al (2011) Highly efficient miRNA-mediated reprogramming of mouse and human somatic cells to pluripotency. Cell Stem Cell 8, 376-388

23. Lin SL, Chang DC, Lin $\mathrm{CH}$, Ying SY, Leu D and Wu DT (2011) Regulation of somatic cell reprogramming through 
inducible mir-302 expression. Nucleic Acids Res 39, 1054-1065

24. Miyoshi N, Ishii H, Nagano H et al (2011) Reprogramming of mouse and human cells to pluripotency using mature microRNAs. Cell Stem Cell 8, 633-638

25. Wang Y, Baskerville S, Shenoy A, Babiarz JE, Baehner L and Blelloch R (2008) Embryonic stem cell-specific microRNAs regulate the G1-S transition and promote rapid proliferation. Nat Genet 40, 1478-1483

26. Wang J, Park JW, Drissi H, Wang X and Xu RH (2014) Epigenetic regulation of miR-302 by JMJD1C inhibits neural differentiation of human embryonic stem cells. J Biol Chem 289, 2384-2395

27. Chang HM, Martinez NJ, Thornton JE, Hagan JP, Nguyen KD and Gregory RI (2012) Trim71 cooperates with microRNAs to repress Cdkn1a expression and promote embryonic stem cell proliferation. Nat Commun 3, 923

28. Cimadamore $\mathrm{F}$, Amador-Arjona $\mathrm{A}$, Chen $\mathrm{C}$, Huang $\mathrm{CT}$ and Terskikh AV (2013) SOX2-LIN28/let-7 pathway regulates proliferation and neurogenesis in neural precursors. Proc Natl Acad Sci U S A 110, E3017-3026

29. Viswanathan SR, Daley GQ and Gregory RI (2008) Selective blockade of microRNA processing by Lin28. Science 320, 97-100

30. Wang G, Guo X, Hong W et al (2013) Critical regulation of miR-200/ZEB2 pathway in Oct4/Sox2-induced mesenchymal-to-epithelial transition and induced pluripotent stem cell generation. Proc Natl Acad Sci U S A 110, 2858-2863

31. Subramanyam D, Lamouille S, Judson RL et al (2011) Multiple targets of miR-302 and miR-372 promote reprogramming of human fibroblasts to induced pluripotent stem cells. Nat Biotechnol 29, 443-448

32. Zhang Z, Hong Y, Xiang D et al (2015) MicroRNA$302 / 367$ cluster governs hESC self-renewal by dually regulating cell cycle and apoptosis pathways. Stem Cell Reports 4, 645-657

33. Barroso-delJesus A, Romero-Lopez C, Lucena-Aguilar G et al (2008) Embryonic stem cell-specific miR302-367 cluster: human gene structure and functional characterization of its core promoter. Mol Cell Biol 28, 6609-6619

34. Rosenberg MI, Georges SA, Asawachaicharn A, Analau E and Tapscott SJ (2006) MyoD inhibits Fstl1 and Utrn expression by inducing transcription of miR-206. J Cell Biol 175, 77-85

35. Rao PK, Kumar RM, Farkhondeh $M$, Baskerville $S$ and Lodish HF (2006) Myogenic factors that regulate expression of muscle-specific microRNAs. Proc Natl Acad Sci U S A 103, 8721-8726

36. Sweetman D, Goljanek K, Rathjen T et al (2008) Specific requirements of MRFs for the expression of muscle specific microRNAs, miR-1, miR-206 and miR-133. Dev Biol 321, 491-499

37. Small EM, O'Rourke JR, Moresi V et al (2010) Regulation of PI3-kinase/Akt signaling by muscle-enriched microRNA486. Proc Natl Acad Sci U S A 107, 4218-4223

38. Yeung F, Chung E, Guess MG, Bell ML and Leinwand LA (2012) Myh7b/miR-499 gene expression is transcriptionally regulated by MRFs and Eos. Nucleic Acids Res 40, 7303-7318
39. Chen JF, Tao Y, Li J et al (2010) microRNA-1 and microRNA-206 regulate skeletal muscle satellite cell proliferation and differentiation by repressing Pax7. J Cell Biol 190, 867-879

40. Kuchen S, Resch W, Yamane A et al (2010) Regulation of microRNA expression and abundance during lymphopoiesis. Immunity $32,828-839$

41. Heo I, Joo C, Kim YK et al (2009) TUT4 in concert with Lin28 suppresses microRNA biogenesis through premicroRNA uridylation. Cell 138, 696-708

42. Hagan JP, Piskounova E and Gregory RI (2009) Lin28 recruits the TUTase Zcchc11 to inhibit let-7 maturation in mouse embryonic stem cells. Nat Struct Mol Biol 16, 10211025

43. Heo I, Ha M, Lim J et al (2012) Mono-uridylation of pre-microRNA as a key step in the biogenesis of group II let-7 microRNAs. Cell 151, 521-532

44. O'Rourke JR, Georges SA, Seay HR et al (2007) Essential role for Dicer during skeletal muscle development. Dev Biol 311, 359-368

45. Davis ME, Zuckerman JE, Choi CH et al (2010) Evidence of RNAi in humans from systemically administered siRNA via targeted nanoparticles. Nature 464, 1067-1070

46. Ma L, Reinhardt F, Pan E et al (2010) Therapeutic silencing of miR-10b inhibits metastasis in a mouse mammary tumor model. Nat Biotechnol 28, 341-347

47. Hansen TB, Jensen TI, Clausen BH et al (2013) Natural RNA circles function as efficient microRNA sponges. Nature 495, 384-388.

48. Memczak S, Jens M, Elefsinioti A et al (2013) Circular RNAs are a large class of animal RNAs with regulatory potency. Nature 495, 333-338

49. Vidigal JA and Ventura A (2015) The biological functions of miRNAs: lessons from in vivo studies. Trends Cell Biol 25, 137-147

50. Rasmussen KD, Simmini S, Abreu-Goodger C et al (2010) The miR-144/451 locus is required for erythroid homeostasis. J Exp Med 207, 1351-1358

51. Medeiros LA, Dennis LM, Gill ME et al (2011) Mir-290295 deficiency in mice results in partially penetrant embryonic lethality and germ cell defects. Proc Natl Acad Sci U S A 108, 14163-14168

52. Kim YK, Wee G, Park J et al (2013) TALEN-based knockout library for human microRNAs. Nat Struct Mol Biol 20, 1458-1464

53. Takada S, Sato T, Ito $Y$ et al (2013) Targeted gene deletion of miRNAs in mice by TALEN system. PLoS One 8 , e76004

54. Xu N, Papagiannakopoulos T, Pan G, Thomson JA and Kosik KS (2009) MicroRNA-145 regulates OCT4, SOX2, and KLF4 and represses pluripotency in human embryonic stem cells. Cell 137, 647-658

55. Lahmy R, Soleimani $M$, Sanati $M H$, Behmanesh $M$, Kouhkan F and Mobarra N (2013) Pancreatic islet differentiation of human embryonic stem cells by microRNA overexpression. J Tissue Eng Regen Med 2013 Jul 30 [Epub ahead of print]

56. Deng S, Zhang Y, Xu C and Ma D (2015) MicroRNA125b-2 overexpression represses ectodermal differentiation of mouse embryonic stem cells. Int J Mol Med 36, 
355-362

57. Lee SW, Yang J, Kim SY et al (2015) MicroRNA-26a induced by hypoxia targets HDAC6 in myogenic differentiation of embryonic stem cells. Nucleic Acids Res 43, 2057-2073

58. Georgantas RW 3rd, Hildreth R, Morisot S et al (2007) CD34 + hematopoietic stem-progenitor cell microRNA expression and function: a circuit diagram of differentiation control. Proc Natl Acad Sci U S A 104, 2750-2755

59. Bruchova H, Yoon D, Agarwal AM, Mendell J and Prchal JT (2007) Regulated expression of microRNAs in normal and polycythemia vera erythropoiesis. Exp Hematol 35, 1657-1667

60. Felli N, Fontana L, Pelosi E et al (2005) MicroRNAs 221 and 222 inhibit normal erythropoiesis and erythroleukemic cell growth via kit receptor down-downmodulation. Proc Natl Acad Sci U S A 102, 18081-18086

61. Wang Q, Huang Z, Xue H et al (2008) MicroRNA miR-24 inhibits erythropoiesis by targeting activin type I receptor ALK4. Blood 111, 588-595

62. Fontana L, Pelosi E, Greco P et al (2007) MicroRNAs 175p-20a-106a control monocytopoiesis through AML1 targeting and M-CSF receptor upregulation. Nat Cell Biol 9, 775-787

63. Zhou B, Wang S, Mayr C, Bartel DP and Lodish HF (2007) miR-150, a microRNA expressed in mature B and T cells, blocks early B cell development when expressed prematurely. Proc Natl Acad Sci U S A 104, 7080-7085

64. Chen CZ, Li L, Lodish HF and Bartel DP (2004) MicroRNAs modulate hematopoietic lineage differentiation. Science 303, 83-86

65. Buckingham M (2006) Myogenic progenitor cells and skeletal myogenesis in vertebrates. Curr Opin Genet Dev $16,525-532$

66. Ivey KN, Muth A, Arnold J et al (2008) MicroRNA regulation of cell lineages in mouse and human embryonic stem cells. Cell Stem Cell 2, 219-229
67. Sluijter JP, van Mil A, van Vliet P et al (2010) MicroRNA-1 and -499 regulate differentiation and proliferation in human-derived cardiomyocyte progenitor cells. Arterioscler Thromb Vasc Biol 30, 859-868

68. Wilson KD, Hu S, Venkatasubrahmanyam S et al (2010) Dynamic microRNA expression programs during cardiac differentiation of human embryonic stem cells: role for miR-499. Circ Cardiovasc Genet 3, 426-435

69. Chen JF, Mandel EM, Thomson JM et al (2006) The role of microRNA-1 and microRNA-133 in skeletal muscle proliferation and differentiation. Nat Genet 38, 228-233

70. Kwon C, Han Z, Olson EN and Srivastava D (2005) MicroRNA1 influences cardiac differentiation in Drosophila and regulates Notch signaling. Proc Natl Acad Sci U S A 102, 18986-18991

71. Wong CF and Tellam RL (2008) MicroRNA-26a targets the histone methyltransferase Enhancer of Zeste homolog 2 during myogenesis. J Biol Chem 283, 9836-9843

72. Sirish P, Lopez JE, Li N et al (2012) MicroRNA profiling predicts a variance in the proliferative potential of cardiac progenitor cells derived from neonatal and adult murine hearts. J Biol Chem 52, 264-272

73. Zhao Y, Samal E and Srivastava D (2005) Serum response factor regulates a muscle-specific microRNA that targets Hand2 during cardiogenesis. Nature 436, 214-220

74. Sun Q, Zhang Y, Yang G et al (2008) Transforming growth factor-beta-regulated miR-24 promotes skeletal muscle differentiation. Nucleic Acids Res 36, 2690-2699

75. Smirnova L, Grafe A, Seiler A, Schumacher S, Nitsch R and Wulczyn FG (2005) Regulation of miRNA expression during neural cell specification. Eur J Neurosci 21, 14691477

76. Visvanathan J, Lee S, Lee B, Lee JW and Lee SK (2007) The microRNA miR-124 antagonizes the anti-neural REST/SCP1 pathway during embryonic CNS development. Genes Dev $21,744-749$ 